\title{
Amiloidosi, ovvero quando le proteine fibrillano. Storia, fisiopatologia e classificazione di una malattia non poi così rara
}

\section{Franco Bergesio}

A nome del Centro Fiorentino per lo studio e la cura delle Amiloidosi, Firenze

\begin{abstract}
Amyloidosis: history, pathophysiology and classification of a not quite so rare disease
Even today amyloidosis is an odd and someway mysterious term for most physicians. Actually we should speak of "Amyloidoses" rather than amyloidosis to indicate a group of protein conformational diseases caused by misfolding and further aggregation of autologous proteins into amyloid fibrils that deposit into organs and tissues. Now more than 30 different proteins have been identified as possible precursors of amyloid fibrils. Regardless of the different precursor protein the misfolding and aggregation process is the same for all the proteins. Here we describe the major mechanisms that lead to fibril formation and eventually cause tissue damage. Finally we elucidate the present classification criteria and differentiate between systemic and localized forms, among which there is Alzheimer's disease. Conversely, major systemic clinical forms are light chain amyloidosis (AL), reactive amyloidosis (AA) and hereditary amyloidosis of which Transthyretin (TTR) is the most common one.

TTR molecule however, even in its wild form (TTRwt), may give rise in older people to amyloid fibrils causing senile systemic amyloidosis (SSA). Kidney is involved in AL, AA and many hereditary forms with the exception of TTR, in which its involvement is extremely rare. Clinical signs of renal amyloidosis largely vary from nephrotic syndrome to different degrees of renal failure according to involvement of different renal anatomical structures.

Keywords: Amyloid fibrils, Immunoglobulin light chain amyloidosis, Protein misfolding, Reactive amyloidosis, Transthyretin amyloidosis
\end{abstract}

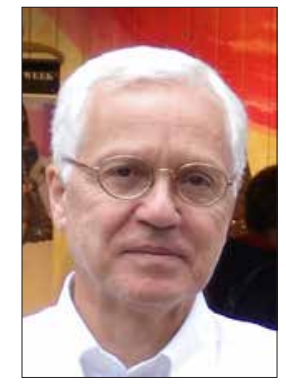

Franco Bergesio

\section{Introduzione}

Ancora oggi il termine Amiloidosi sembra rimanere avvolto da un alone di mistero e di rarità che tuttavia gli studi e le ricerche degli ultimi 20-30 anni hanno cercato di dissipare, riuscendoci in gran parte ma solo per una ristretta cerchia di addetti ai lavori.

Per oltre un secolo la malattia è rimasta una semplice curiosità per il pa-

Accepted: February 18, 2017

Published online: May 16, 2017

Indirizzo per la corrispondenza:

Franco Bergesio

Centro Fiorentino per lo studio e la cura delle Amiloidosi

Via del Pino 17

50137 Firenze

www.amiloidosifirenze.it

francobergesio@gmail.com tologo e una malattia misteriosa e incurabile per il clinico. Questo non è più vero oggi che ne conosciamo la natura e i meccanismi del danno e disponiamo finalmente di terapie sempre più efficaci.

La storia dell'Amiloidosi è un po' la storia delle proteine e di come queste possono assumere conformazioni diverse a cui corrispondono funzioni e ruoli biologici diversi.

Nella maggior parte dei casi esse sono ripiegate secondo una struttura globulare ad alfa elica con la quale esercitano le loro specifiche funzioni biologiche: enzimatiche, di sostegno, plastiche e così via.

In alcune condizioni però esse possono perdere la loro normale conformazione globulare dovuta al ripiegamento o "folding" di parti della catena polipeptidica per dispiegarsi ("unfolding") e assumere un tipico aspetto a "foglietto beta" con perdita delle loro funzioni biologiche e tendenza ad aggregarsi in protofilamenti (2 catene polipeptidiche adiacenti) e poi in fibrille amiloidi (4-6 protofilamenti) all'interno degli spazi extracellulari di organi e tessuti.

Esistono tuttavia in natura delle proteine la cui conformazione "normale", quella cioè con cui svolgono la loro abituale funzione biologica, è quella del foglietto beta, come, per esempio, la proteina della tela del ragno o quella caratteristica della seta. 


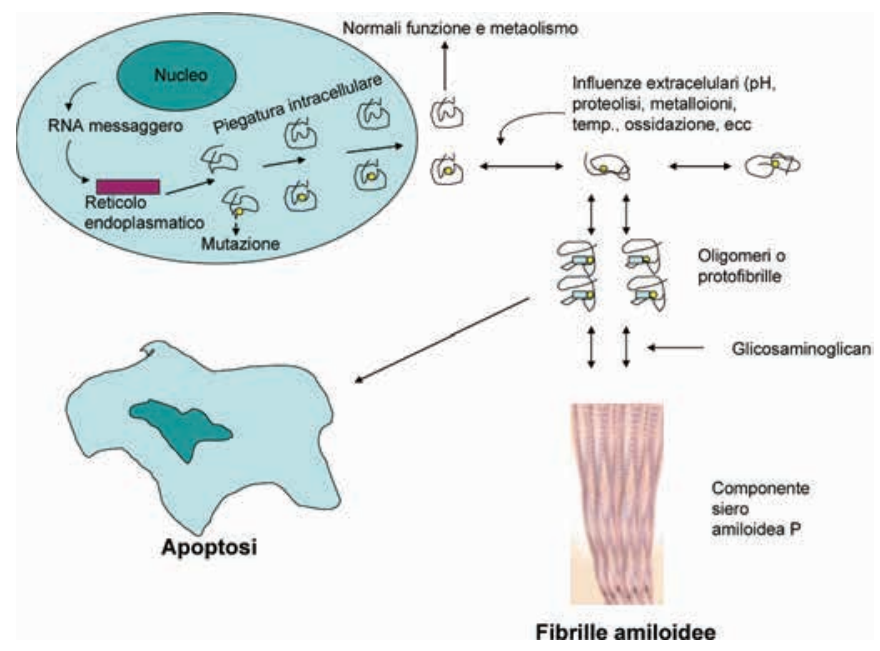

Fig. 1 - Destino metabolico di una catena polipeptidica appena sintetizzata in un paziente eterozigote per una mutazione della Transtiretina (TTR). Le catene polipeptidiche della TTR vengono sintetizzate nel reticolo endoplasmatico, ripiegate ("folding intracellulare") e, una volta superato il controllo di qualità, vengono secrete nello spazio extracellulare. Qui le molecole mutate possono passare dalla corretta forma ripiegata nativa a una parzialmente ripiegata in equilibrio con la precedente. Le proteine normali (native) sono funzionalmente attive e sono normalmente metabolizzate. Quelle parzialmente ripiegate possono ritornare a essere normalmente ripiegate o generare molecole mal ripiegate o "misfolded" che hanno un'elevata propensione ad auto aggregarsi in oligomeri o protofilamenti. Particolari condizioni ambientali e modificazioni chimiche possono favorire il percorso patologico. Oligomeri e protofilamenti possono avere una tossicità cellulare attraverso un meccanismo che attiva l'apoptosi e, con l'aiuto di costituenti comuni come i glicosaminoglicani (GAG), dare luogo alla formazione di fibrille amiloidi.

\section{Fisiopatologia}

Fisiologicamente le proteine appena sintetizzate all'interno delle cellule subiscono rapide modificazioni conformazionali che fanno assumere loro l'aspetto naturale globulare prima di essere rilasciate nello spazio extracellulare. Questo processo di ripiegamento o "folding" è un processo dinamico che attraverso passaggi intermedi porta le proteine ad assumere la loro conformazione naturale a cui è legata la loro funzione biologica oppure, in una parte, a mal ripiegarsi "misfolded" per poi aggregarsi, una volta secrete nello spazio extracellulare, in protofilamenti e fibrille (Fig. 1) (1).

Durante questo processo le proteine sono sottoposte a un controllo di qualità per cui solo quelle idonee, con la conformazione adeguata ("folded"), vengono secrete, mentre le altre vengono eliminate dai proteasomi (2).

Una volta secreta, la proteina mantiene un equilibrio dinamico con le forme parzialmente ripiegate che, in condizioni ambientali favorevoli, possono evolvere verso l'aggregazione in fibrille amiloidi (Fig. 1).
Diversi sono i meccanismi che favoriscono Il processo amiloidogenico:

- la proteina, pur avendo struttura e concentrazione normali, ha un'intrinseca propensione ad assumere conformazioni patologiche (unfolded) che si rende più evidente con il processo dell'invecchiamento. È il caso della molecola naturale della Transtiretina (TTRwt) nelle forme di Amiloidosi sistemica senile (SSA);

- la proteina, strutturalmente normale, è presente nel sangue/tessuti a concentrazioni molto elevate per molto tempo: è il caso della Beta2microglobulina dei pazienti in dialisi cronica e della Siero Amiloide A (SAA) dei pazienti affetti da processi infiammatori cronici. Nel primo caso si parla di Amiloidosi Dialitica e nel secondo di Amiloidosi Reattiva o Secondaria (AA);

- un terzo meccanismo consiste nella presenza di proteine anomale a causa di una mutazione che comporta la sostituzione di un singolo aminoacido all'interno della catena polipeptidica, cosa che rende la proteina, in taluni casi, fortemente instabile e amiloidogenica. Questo meccanismo è caratteristico delle forme di Amiloidosi ereditaria. Tra queste, la più comune è l'amiloidosi da mutazione della molecola della TTR (TTRm) di cui a oggi si riconoscono oltre 100 mutazioni amiloidogeniche. Altre forme ereditarie riguardano la molecola del Lisozima e quella della catena alfa del Fibrinogeno $A$, dell'Apolipoproteina A1 e così via;

- un ulteriore meccanismo è il riarrangiamento proteolitico del precursore proteico come nel caso della proteina ß-amiloide nell'Alzheimer.

Questi meccanismi possono agire indipendentemente o in associazione l'uno con l'altro, come, per esempio, nel caso delle catene leggere immunoglobuliniche $(\mathrm{CL})$, dove le fisiologiche mutazioni della parte variabile delle $\mathrm{CL}$ unite talora alle elevate concentrazioni o ad altri fattori locali contribuiscono al processo di amiloidogenesi (1).

Le fibrille di Amiloide presentano tutte, indipendentemente dalla proteina precursore, ovvero dal tipo di amiloidosi, una struttura secondaria a foglietto beta e dei comuni costituenti quali la $S A P$, che conferisce stabilità alla molecola, i Proteoglicani e l'Apolipoproteina E. Questa uguale composizione conferisce alle fibrille la comune caratteristica di assumere una colorazione verde mela se osservate in luce polarizzata dopo colorazione con Rosso Congo, caratteristica fondamentale per la loro identificazione e quindi per la diagnosi della malattia.

In ME, le fibrille hanno un aspetto rigido e non ramificato, con un diametro di 7,5-10 nm (Fig. 2).

\section{Meccanismi del danno tissutale}

A oggi si riconoscono due principali meccanismi di danno tissutale in corso di amiloidosi. 


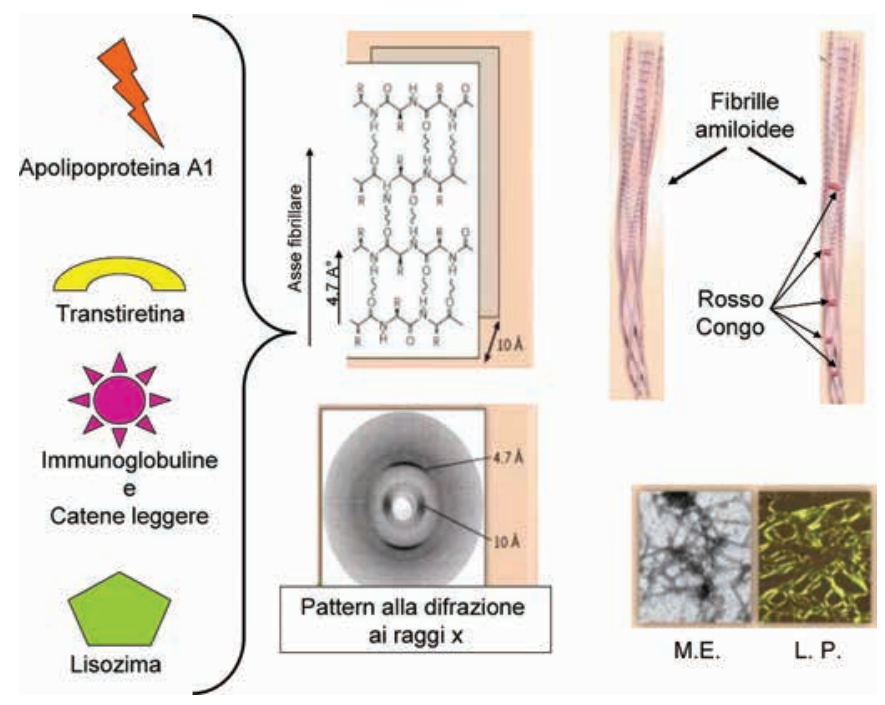

Fig. 2 - Aspetti Strutturali dell'Amiloide. Sulla sinistra ci sono le 4 molecole tridimensionali della Transtiretina, Apolipoproteina A1, Lisozima e catena leggera $\mathrm{k}$ delle Immunoglobuline che convergono tutte a formare la tipica struttura a foglietto ß (al centro) con il caratteristico pattern di diffrazione ai raggi x. La struttura originale del precursore non è più riconoscibile in questa fase. Due foglietti ß contigui costituiscono un protofilamento. Come è mostrato a destra, 4-6 protofilamenti si legano insieme I'un l'altro per formare una fibrilla amiloide con un caratteristico diametro di 7.5-10 nm. Questa struttura consente la deposizione a intervalli regolari del colorante Rosso Congo, cosa che conferisce la tipica proprietà tintoriale dell'amiloide quando osservata in Microscopia Ottica a luce polarizzata.

Uno è il danno meccanico provocato dall'accumulo di fibrillenellospazio extracellulare con progressiva dislocazione e compressione dei tessuti circostanti e l'altro è il danno tossico diretto esercitato dagli oligomeri e dai protofilamenti originatisi dai precursori amiloidogenici durante il processo di fibrillogenesi. Questo fenomeno è stato evidenziato e studiato soprattutto per quelle forme originatesi dalle catene leggere libere delle Ig, ma sembra operante anche per altre molecole, come, per esempio, la TTR mutata o naturale.

La tossicità miocardica diretta ad opera delle catene leggere è stata ben evidenziata e documentata dagli esperimenti in vivo sul verme $C$. Elegans, la cui vitalità e attività peristaltica del faringe (evolutivamente assimilabile al cuore dei vertebrati) sono marcatamente inibite e ridotte dalla concentrazione delle $\mathrm{CL}$ amiloidogeniche presenti nel terreno di coltura (3).

\section{Classificazione}

Tutti i depositi di amiloidosi hanno caratteristiche simili, tuttavia i precursori proteici da cui derivano sono molto diversi e diversi sono i meccanismi fisiopatologici che li hanno prodotti e dunque diversi saranno gli approcci terapeutici per ciascun tipo di amiloidosi.

Questo fa sì che non parliamo di amiloidosi al singolare ma di amiloidosi al plurale. Ciascuna forma è identificata dalla lettera A di amiloidosi seguita dall'iniziale della proteina precursore delle fibrille, per esempio $\mathbf{A L}=\mathbf{A}$ per Amiloidosi e $\mathbf{L}$ per Light chain, la catena leggera immunoglobulinica, $\mathbf{A A}=$ A per Amiloidosi e A per SAA, siero amiloide A, nome di una proteina della fase acuta dell'infiammazione.

Attualmente sono state identificate oltre 30 differenti proteine che possono causare amiloidosi.

I depositi di amiloidosi possono essere sistemici o localizzati, come nelle forme di amiloidosi cerebrale, di cui la più comune è la malattia di Alzheimer, e nelle forme localizzate di amiloidosi AL, che interessano soprattutto le vie aeree, la cute e le vie urinarie e che di solito non richiedono una terapia sistemica.

Nella Tabella I sono riportate alcune delle principali forme di amiloidosi, il loro precursore, la distribuzione sistemica o localizzata e il quadro clinico al quale sono associate. Evidenziate in giallo le più importanti forme con interessamento renale.

Riquadrate in rosso e in ordine di frequenza le forme più comuni di amiloidosi renale con l'eccezione dell'ATTR, in cui l'interessamento renale è estremamente raro.

Ritenuta una malattia rara e inserita nell'elenco nazionale e regionale delle malattie rare, la malattia è stata diagnosticata negli ultimi anni con sempre maggiore frequenza e le nuove diagnosi nel territorio dell'area fiorentina (ex provincia di Firenze) negli anni 2015 e 2016 sono arrivate al numero di 45-50 per anno per milione di abitanti con le AL intorno a 15-16 casi/anno, rimanendo le Amiloidosi nell'ambito delle patologie rare, ma non così rare come eravamo abituati a considerarle fino a qualche anno fa.

\section{Le Amiloidosi Renali}

Diverse sono le forme di amiloidosi che possono coinvolgere il rene. In ordine di frequenza le $\mathbf{A L}$, dove il rene insieme al cuore è l'organo più frequentemente interessato, e le forme secondarie o AA, dove il rene è l'organo in assoluto più colpito. In entrambe queste forme il danno renale si manifesta, nella stragrande maggioranza dei casi, con una proteinuria generalmente nefrosica, con o senza un'insufficienza renale.

Il rene è poi interessato da numerose ma rare forme eredofamiliari, tra cui le Amiloidosi da ApoA1, da ApoA2 e quella da Gelsolina, da catena alfa del Fibrinogeno A, Lisozima, ApoC2 e ApoC3.

È invece estremamente raro e tardivo il coinvolgimento renale legato a mutazioni della molecola della TTR (vedi forme evidenziate in Tab. I).

Infine dobbiamo ricordare l'ALECT2, legata al fattore chemotattico leucocitario 2, e l'ApoA4, le cui origini restano ancora sconosciute. Tutte queste forme mostrano generalmente 
TABELLA I - Principali tipi di amiloidosi e loro precursore proteico

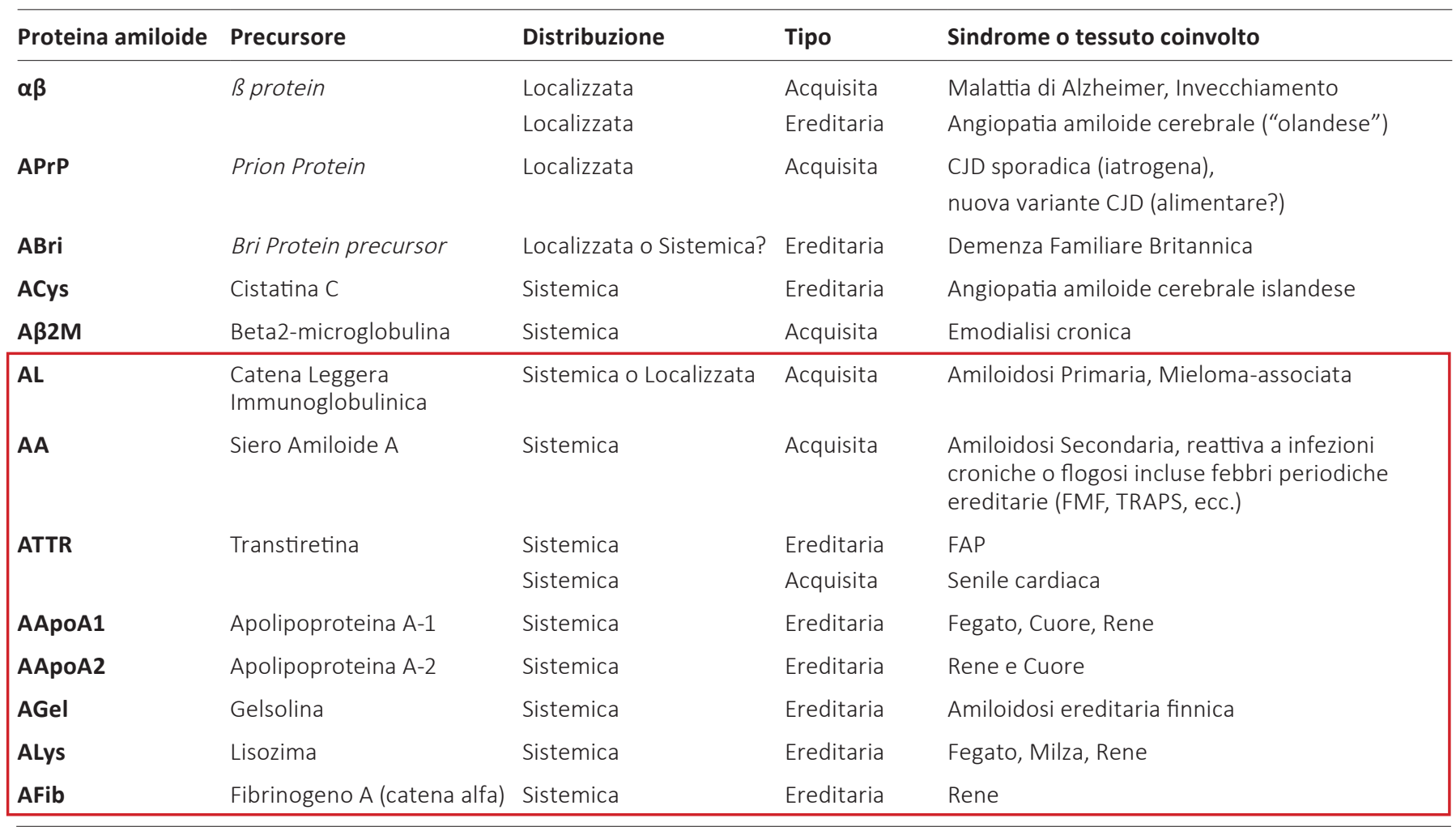

un coinvolgimento della corticale, soprattutto dei glomeruli ma anche dell'interstizio e dei tubuli, con un quadro clinico caratterizzato da una sindrome nefrosica con o senza insufficienza renale. Diversamente le forme da ApoA1 e ApoA4 mostrano un esclusivo interessamento della midollare con un quadro clinico di insufficienza renale a lenta evoluzione (4).

\section{Disclosures}

Financial support: No financial support was received for this submission.

Conflict of interest: The authors have no conflict of interest.

\section{Bibliografia}

1. Merlini G, Bellotti V. Molecular mechanisms of Amyloidosis. N.Eng J Med. 2003;349:583-596.

2. Sitia R, Palladini G, Merlini G. Bortezomib in the treatment of AL-amyloidosis: targeted therapy? Haematologica. 2007; 92(10):1302-1307.

3. Diomede L, Rognoni P, Lavatelli F, et al. Caenorhabditis elegansbased assay recognizes immunoglobulin light chains causing heart amyloidosis. Blood. 2014;123:3543-3552.

4. Obici L, Nuvolone M, Merlini G. Expanding the spectrum of systemic amyloid diseases: a new hint from the kidney. Kidney Int. 2016;90:479-481.

\section{TEST DI VERIFICA}

\section{1) L'amiloidosi è una malattia:}

a) Dovuta a un'alterata conformazione delle proteine coinvolte

b) Dovuta a una mutazione di un singolo acido della proteina coinvolta

c) Ematologica secondaria a una patologia plasma cellulare

d) In cui il rene è spesso coinvolto.

\section{2) II coinvolgimento renale nell'amiloidosi:}

a) Si manifesta sempre con una sindrome nefrosica con o senza insufficienza renale

b) È caratterizzato comunemente da una sindrome nefrosica ma talora solo da un'insufficienza renale di origine sconosciuta

c) È raro nelle forme secondarie e comune nelle $\mathrm{AL}$

d) È sempre caratterizzato da insufficienza renale. 